CARDIOVASCULAR MEDICINE

\title{
Effect of one or more co-morbid conditions on diagnostic accuracy of coronary flow velocity reserve for detecting significant left anterior descending coronary stenosis
}

\author{
H Yoshitani, M Takeuchi, K Sakamoto, T Akasaka, K Yoshida, J Yoshikawa
}

Heart 2005;91:1294-1298. doi: 10.1136/hrt.2004.042549

\begin{abstract}
See end of article for authors' affiliations

Correspondence to

Dr Masaaki Takeuchi,

Department of Internal

Medicine, Tane General

Hospital, 1-2-31

Sakaigawa, Nishi-ku,

Osaka 550-0024 Japan;

masaaki_takeuchi@

hotmail.com
\end{abstract}

Accepted 29 October 2004

\begin{abstract}
Objective: To determine the effect of one or multiple co-morbid conditions on the diagnostic accuracy of coronary flow velocity reserve (CFVR) in a heterogeneous patient population.

Methods: CFVR was measured in the left anterior descending coronary artery (LAD) by transthoracic Doppler echocardiography (TTDE) in 318 consecutive patients before elective coronary angiography. CFVR was calculated as the average peak diastolic velocity during intravenous ATP infusion divided by baseline flow velocity. All patients underwent coronary angiography within 48 hours. Significant LAD stenosis was defined as $>50 \%$ luminal narrowing. Diagnostic accuracy of CFVR was analysed according to the type and number of risk factors that may adversely affect microvascular function.

Results: CFVR was measured in 309 patients, of whom 105 were found to have significant LAD stenosis based on coronary angiography. CFVR $<2.0$ had a sensitivity of $86 \%$ and a specificity of $77 \%$ for predicting significant LAD stenosis. Left ventricular hypertrophy (LVH) was the only factor that significantly lowered diagnostic accuracy $161 \%$ with LVH $v 84 \%$ without $\mathrm{LVH}, \mathrm{p}<0.001)$. Diagnostic accuracy was not affected by increasing number of risk factors.

Conclusions: The diagnostic accuracy of CFVR by TTDE for detecting significant LAD stenosis remains high in a more clinically relevant population with multiple cardiovascular co-morbidities. Only the presence of LVH adversely affected diagnostic accuracy.
\end{abstract}

A lthough coronary angiography is still the ideal means of diagnosing coronary artery disease, visual estimation of the anatomical severity of coronary artery stenosis is not always accurate for determining its physiological importance, even when using quantitative analysis. ${ }^{1-3}$ Coronary flow reserve or coronary flow velocity reserve (CFVR) is a useful index for predicting physiologically significant coronary stenosis. Historically, it has been measured by one of three methods: an invasive intracoronary Doppler guidewire, ${ }^{45}$ semi-invasive transoesophageal Doppler echocardiography, ${ }^{6-8}$ or expensive positron emission tomography. ${ }^{9}$ However, recent developments in echocardiographic technology enable the non-invasive assessment of CFVR by means of transthoracic Doppler echocardiography (TTDE). Several previous studies have reported the feasibility and accuracy of CFVR measurements in the left anterior descending coronary artery (LAD) by TTDE..$^{10-13}$ The value of CFVR depends on the severity of the epicardial coronary stenosis as well as the status of microvascular function. Thus, in the presence of factors that may adversely affect microvascular function, such as hypertension, diabetes, hypercholesterolaemia, smoking, left ventricular hypertrophy $(\mathrm{LVH})$, and old myocardial infarction, CFVR may be abnormal even in the absence of a significant epicardial coronary stenosis. ${ }^{14-20}$ Previous studies validating CFVR by TTDE were derived from highly selected populations; therefore, their results could not be extrapolated to daily clinical practice, where patients are known to have multiple instead of single cardiovascular co-morbid conditions. ${ }^{10} 21-23$ Few studies have addressed the effects of coronary risk factors on CFVR. ${ }^{24}$ In light of this, we undertook this study to evaluate the effect of having one or multiple risk factors on the diagnostic accuracy of CFVR for detecting significant stenosis of the LAD in a large cohort of patients undergoing coronary angiography.

\section{METHODS}

Study patients

We prospectively enrolled 318 consecutive patients (224 men and 94 women; mean (SD) age 66 (10) years, range 34-89 years) who were scheduled for elective coronary angiography. Coronary angiography was performed because of suspected or known coronary artery disease. Patients with unstable angina, intractable heart failure, and severe valvar heart disease were excluded. The study protocol was approved by the institutional review boards of Tane General Hospital. Informed consent was obtained from all patients before the study. We examined six factors that may affect the coronary microcirculation: hypertension, hypercholesterolaemia, diabetes, smoking, LVH, and previous myocardial infarction. Each factor was defined as follows. Hypertension was defined as blood pressure $>140 / 85 \mathrm{~mm} \mathrm{Hg}$ on repeated measurements or if the patient was taking antihypertensive medications. Hypercholesterolaemia was defined as total cholesterol concentration $>5.7 \mathrm{mmol} / \mathrm{l}$ or if the patient was taking cholesterol lowering medications. Diabetes was defined as fasting glucose concentration $>6.99 \mathrm{mmol} / \mathrm{l}$ or if the patient was taking hypoglycaemic agents. Patients were considered to be smokers if they had stopped less than three months previously or were current smokers. Previous anterior myocardial infarction was defined by an ECG abnormality or past angiographic records. For LVH determination, left ventricular mass and left ventricular mass index were calculated in accordance with Devereux's formula, and LVH was defined as a left ventricular mass index $>125 \mathrm{~g} / \mathrm{m}^{2}$ (men) or $>120 \mathrm{~g} / \mathrm{m}^{2}$ (women) as previously reported..$^{25}$

Abbreviations: APDV, average peak diastolic velocity; $B$, unstandardised regression coefficient; CFVR, coronary flow velocity reserve; $L A D$, left anterior descending coronary artery; $L V H$, left ventricular hypertrophy; TTDE, transthoracic Doppler echocardiography 


\section{Coronary flow velocity measurement}

The detail of coronary flow velocity measurement by TTDE has been described elsewhere..$^{7011} 13$ If the colour Doppler signal of the LAD was not readily obtained or if the Doppler spectral signal of the LAD flow was not clear, $0.5-1 \mathrm{ml}$ of Levovist $(300 \mathrm{mg} / \mathrm{ml}$, Tanabe Seiyaku, Inc, Tokyo, Japan and Schering AG, Berlin, Germany) was administered intravenously to enhance the Doppler flow signal of the LAD.

\section{Coronary angiography}

Coronary angiography was performed by the Judkins or Sones technique with standard equipment. Coronary stenosis was evaluated from multiple projections by an experienced investigator who was unaware of CFVR data. The severity of coronary stenosis was visually determined and expressed as a percentage of the lumen diameter. Any stenosis in the LAD $>50 \%$ was considered significant.

\section{Study protocol}

After baseline coronary flow velocity was recorded in the distal LAD, ATP was intravenously infused ( $140 \mu \mathrm{g} / \mathrm{kg} / \mathrm{min})$ for three minutes to record coronary flow velocity during maximum hyperaemia. The ECG and heart rate were monitored continuously during the study. Blood pressure was measured at baseline and once every minute during intravenous ATP infusion. The position and angle of the transducer on the chest wall were maintained before, throughout, and after the administration of ATP. Each study was performed by one experienced investigator who was blinded to the patient's medical history and indications for coronary angiography. The record of the Doppler signal was analysed off line by use of the built-in calculation package in the ultrasound unit. The spectral Doppler signals of one representative cardiac cycle at baseline and during hyperaemia were selected. The diastolic flow envelope was traced, generating the time averaged peak diastolic velocity (APDV) at baseline and during maximum hyperaemia. CFVR was calculated as APDV during maximum hyperaemia divided by baseline APDV. CFVR > 2.0 was defined as normal, based on the results of previous studies that evaluated flow velocities in the distal LAD. ${ }^{1021-23}$ All patients underwent coronary angiography less than 48 hours after the measurement of CFVR. Intraobserver and interobserver variability of CFVR by TTDE, having been previously assessed at our institution, were $5.0 \%$ and $7.5 \%$, respectively. ${ }^{26}$ This is well within the limits of clinical acceptability.

\section{Statistical analysis}

Data are expressed as mean (SD). Parametric data between two groups were analysed by paired or unpaired $t$ tests. For comparisons of sensitivity, specificity, and accuracy of the methods for detection of stenosis of LAD between groups, we used Fisher's exact test, Mann-Whitney U test, and $\chi^{2}$ test for univariate test, when appropriate. A probability value of $\mathrm{p}<0.05$ was considered significant. We examined the

\begin{tabular}{ll} 
Table 1 Demographic and clinical features \\
\hline Age (years) & $66(10)$ \\
Men/women & $216 / 93$ \\
Risk factors & \\
Hypertension & $196(63 \%)$ \\
Hypercholesterolaemia & $160(52 \%)$ \\
Diabetes & $139(45 \%)$ \\
Current smoker & $106(34 \%)$ \\
Prior anterior MI & $74(24 \%)$ \\
LVH & $54(17 \%)$ \\
\hline
\end{tabular}

Data are mean (SD) or number (\%).

LVH, left ventricular hypertrophy; MI, myocardial infarction. sensitivity and specificity of CFVR by TTDE for predicting significant LAD stenosis across a range of cut off values. A receiver operating characteristic curve was created to define the optimum cut off value. Multiple linear regression analysis was used to test for independent associations between CFVR and various factors. Calculations were made with the use of the StatView version 5.0 statistical software (SAS Institute, Cary, North Carolina, USA).

\section{RESULTS}

\section{Patient characteristics}

The quality of the spectral Doppler coronary flow envelope both at baseline and during maximum hyperaemia was adequate in 309 of 318 patients (97\%). Echocardiographic contrast agent was required for 155 patients (50\%) to improve the spectral Doppler signals. Table 1 lists clinical characteristics and angiographic data of these 309 patients. Coronary angiography showed significant LAD stenosis in 105 patients, including 10 with total occlusion in the proximal LAD. In 54 patients with LVH, 39 patients had hypertension and 10 had prior myocardial infarction. Five patients had no definite cause of LVH.

\section{Haemodynamic data}

No patient developed adverse effects, such as hypotension, atrioventricular block, or severe chest pain, during the administration of ATP. Heart rate increased from 69 (11) beats/min to $74(12)$ beats/min $(p<0.001)$. Systolic and diastolic blood pressure decreased from 134 (23) $\mathrm{mm} \mathrm{Hg}$ to 124 (22) $\mathrm{mm} \mathrm{Hg}$ and from 76 (12) $\mathrm{mm} \mathrm{Hg}$ to 71 (13) $\mathrm{mm} \mathrm{Hg}(p<0.001)$, respectively. There were no significant differences in response to ATP infusion in terms of heart rate, systolic blood pressure, and diastolic blood pressure between patients with and those without significant stenosis in the LAD.

\section{CFVR}

Baseline APDV did not differ between patients with significant LAD stenosis and those without LAD stenosis $(17.8(9.7) \mathrm{cm} / \mathrm{s} \vee 18.6(7.2) \mathrm{cm} / \mathrm{s}$, respectively). However, APDV during hyperaemia was significantly lower in patients with significant LAD stenosis than in those with no LAD stenosis $(25.8(15.8) \mathrm{cm} / \mathrm{s} \vee 44.2(16.0) \mathrm{cm} / \mathrm{s}, \mathrm{p}<0.001)$. Similarly, CFVR differed significantly between the two groups (1.44 (0.58) v 2.52 (0.79), p < 0.001). No significant difference of CFVR was noted between patients with prior myocardial infarction in the LAD territory and those without $(2.04(0.97) v 2.19(0.86))$, between patients with hypertension and those without $(2.14(0.86) v 2.19(0.94))$, between patients with hypercholesterolaemia and those without (2.19 $(0.85) \vee 2.12(0.93))$, and between patients with LVH and those without $(1.98(0.75) v 2.19(0.91))$. However, CFVR was significantly lower in patients with diabetes than in those without $(2.00(0.92) v 2.28(0.85), \mathrm{p}<0.005)$. The prevalence of LAD disease was significantly higher in diabetic patients than in those without $(40 \% v 29 \%, \mathrm{p}<0.05)$. No significant difference of CFVR was noted between diabetic patients with good control (haemoglobin Alc $<8 \%$ ) and those with poor control (haemoglobin Alc $\geqslant 8 \%)(2.03$ (0.88) v 1.83 (1.12), $\mathrm{p}=0.07$ ).

Applying a cut off value of CFVR $<2.0$ to the entire population had a sensitivity of $86 \%$, a specificity of $77 \%$, and a diagnostic accuracy of $80 \%$ for predicting significant LAD stenosis. Next, we examined the influence of six factors, hypertension, hypercholesterolaemia, diabetes, smoking, $\mathrm{LVH}$, and prior myocardial infarction in the LAD territory, on the accuracy of predicting significant LAD stenosis. Table 2 shows the effect of each factor individually on the diagnostic accuracy of CFVR. A significant reduction of diagnostic 
Table 2 Sensitivity and specificity for predicting significant left anterior descending coronary artery (LAD) stenosis with CFVR $<2.0$

\begin{tabular}{lllllll}
\hline & Number & Sens (\%) & Spec (\%) & PPV (\%) & NPV (\%) & Acc (\%) \\
\hline Total & 309 & 86 & 77 & 66 & 91 & 80 \\
Hypertension +/- & $196 / 113$ & $84 / 89$ & $78 / 77$ & $67 / 64$ & $90 / 94$ & $80 / 81$ \\
Hypercholesterolaemia +/- & $160 / 149$ & $84 / 88$ & $83 / 72$ & $73 / 60$ & $90 / 92$ & $84 / 77$ \\
Diabetes +/- & $139 / 170$ & $86 / 86$ & $75 / 79$ & $70 / 63$ & $91 / 93$ & $79 / 81$ \\
Smoking +/- & $106 / 203$ & $91 / 83$ & $74 / 79$ & $61 / 69$ & $95 / 90$ & $79 / 81$ \\
Prior anterior MI+/- & $74 / 235$ & $88 / 85$ & $74 / 78$ & $72 / 64$ & $89 / 92$ & $80 / 80$ \\
LVH +/- & $54 / 255$ & $79 / 87$ & $55 / 83^{*}$ & $38 / 74^{* *}$ & $88 / 92$ & $61 / 84^{* *}$ \\
\hline
\end{tabular}

${ }^{*} \mathrm{p}<0.005 ;{ }^{* *} \mathrm{p}<0.001$

Acc, accuracy; CFVR, coronary flow velocity reserve; NPV, negative predictive value; PPV, positive predictive value; Sens, sensitivity; Spec, specificity.

accuracy was observed only in patients with LVH compared with those without LVH $(61 \% v 84 \%, \mathrm{p}<0.001)$. Figure 1 shows an example of a patient with LVH. To determine the optimum cut off point for the diagnostic accuracy of CFVR in patients with LVH, we performed receiver operating characteristic analysis (table 3 ). We found that a cut off value of CFVR $<1.5$ provided the best diagnostic accuracy (sensitivity $64 \%$, specificity $88 \%$, and accuracy $81 \%$ ) in the setting of LVH.

Table 4 shows the effect of the number of risk factors on the diagnostic accuracy of CFVR. Thirteen patients had no risk factors, 62 had one, 103 had two, 76 had three, and 55 had four or more factors that may affect microvascular function. Sensitivity was not changed significantly among the groups. Specificity and diagnostic accuracy tended to decrease as the number of risk factors increased but no significant difference was noted.

Multivariate linear regression analysis of the study objectives showed that the value of CFVR in the LAD was associated with the presence of LAD stenosis angiographically (unstandardised regression coefficient $(\mathrm{B})=-1.070$, $p<0.0001)$, heart rate at rest $(B=-0.014, p=0.0002)$, presence of LVH $(\mathrm{B}=-0.322, \mathrm{p}=0.0033)$, and age $(\mathrm{B}=-0.009, \mathrm{p}=0.0384)($ table 5$)$.

\section{DISCUSSION}

This study reinforced the high feasibility of CFVR measurements and its acceptable diagnostic accuracy for the detection of significant LAD stenosis by TTDE in a relatively large, heterogeneous patient population. Although most patients had one or more factors that would adversely affect coronary microvascular function, only the presence of LVH significantly decreased the diagnostic accuracy of CFVR. This suggests that only in the presence of LVH should alternative criteria be used to interpret CFVR results.

\section{Previous studies}

Measurement of CFVR provides useful information for detecting functionally significant coronary artery stenosis. ${ }^{10-}$ ${ }^{13}$ However, its calculation depends on not only the severity of proximal epicardial coronary stenosis but also microvascular function distal to the recording site. Impaired coronary flow velocity or CFVR has been reported in patients with hypertension, hypercholesterolaemia, diabetes, and LVH
A

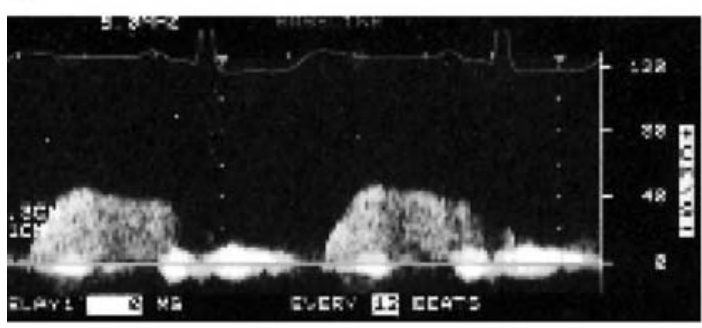

B

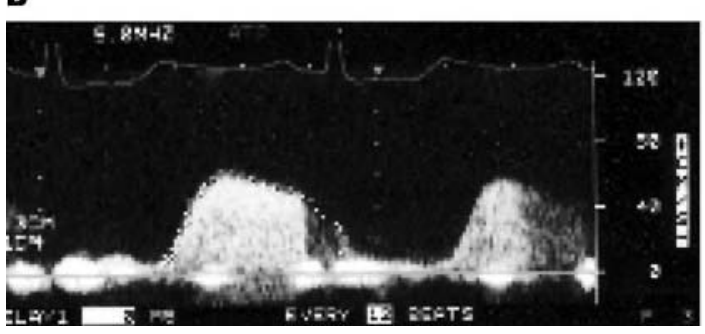

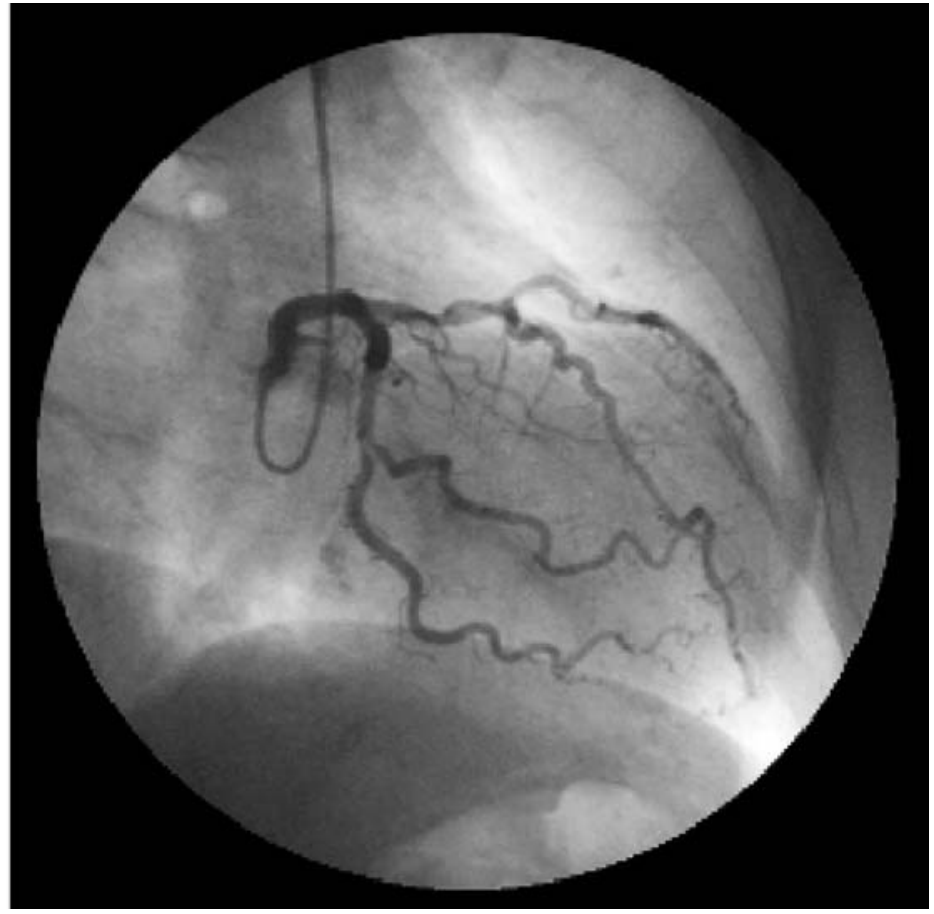

Figure 1 Coronary flow velocity in the distal left anterior descending coronary artery (LAD) (A) at baseline and (B) during ATP infusion in a patient with left ventricular hypertrophy. A blunted increase in the coronary flow velocity during ATP infusion resulted in low coronary flow velocity reserve of 1.3. (C) Coronary angiography showed no significant stenosis in the LAD. 
Table 3 Diagnostic value of CFVR in patients with LVH

\begin{tabular}{llllll}
\hline CFVR cut off & Sens $(\%)$ & Spec (\%) & PPV (\%) & NPV (\%) & Acc (\%) \\
\hline 2.1 & 79 & 48 & 34 & 86 & 56 \\
2.0 & 79 & 58 & 37 & 85 & 61 \\
1.9 & 71 & 58 & 37 & 85 & 61 \\
1.8 & 71 & 60 & 38 & 86 & 63 \\
1.7 & 71 & 63 & 40 & 86 & 65 \\
1.6 & 71 & 78 & 53 & 89 & 76 \\
1.5 & 64 & 88 & 64 & 88 & 81 \\
1.4 & 50 & 90 & 64 & 84 & 80 \\
\hline
\end{tabular}

and in smokers. ${ }^{14-20}$ CFVR may also be depressed in patients with previous myocardial infarction even in the absence of significant infarct related artery stenosis. Although previous validation studies have reported high sensitivity and specificity of CFVR in the LAD assessed by TTDE for detecting angiographically significant LAD stenosis, patients with hypertension, diabetes, and previous myocardial infarction were excluded from the study populations. ${ }^{21-23}$ Thus, these data cannot be extrapolated to the general population. Recent CFVR studies with dipyridamole stress echocardiography have enrolled more heterogeneous populations, and their respective diagnostic accuracies are in agreement with our overall results. ${ }^{27}{ }^{28}$ However, these studies did not specifically address the possible effects of one or multiple risk factors on the diagnostic accuracy of CFVR.

\section{Current study}

In this study, we enrolled a more clinically relevant population, in contrast to prior studies of CFVR. Highlighting this is the presence in three quarters of the study patients of two or more risk factors that may adversely affect coronary microvascular function. Even in this heterogeneous group, CFVR $<2.0$ provided acceptable diagnostic value $(86 \%$ sensitivity and $77 \%$ specificity) for detecting significant LAD stenosis. We recognise that these values are somewhat lower than those in previous validation studies applied to a more homogeneous patient population with respect to cardiovascular risk factors. However, when the 54 patients with LVH are excluded, the sensitivity and specificity improves to $87 \%$ and $83 \%$, respectively. In addition, although this analysis shows a trend towards decreasing specificity and accuracy as the number of risk factors increase, there was no significant difference between these groups. In summary, only in patients with LVH did we observe significantly lower specificity $(55 \%)$ and diagnostic accuracy $(61 \%)$ at a cut off
Table 4 Diagnostic accuracy according to the number of risk factors

\begin{tabular}{llll}
\hline $\begin{array}{l}\text { Risk } \\
\text { factors }\end{array}$ & Sens $(\%)$ & Spec $(\%)$ & Acc $(\%)$ \\
\hline 0 & $75(3 / 4)$ & $78(7 / 9)$ & $77(10 / 13)$ \\
1 & $89(16 / 18)$ & $84(37 / 44)$ & $85(53 / 62)$ \\
2 & $86(31 / 36)$ & $81(54 / 67)$ & $83(85 / 103)$ \\
3 & $87(19 / 21)$ & $72(35 / 53)$ & $72(55 / 76)$ \\
$\geqslant 4$ & $83(20 / 24)$ & $71(22 / 31)$ & $76(42 / 55)$ \\
\hline
\end{tabular}

value of $\mathrm{CFVR}<2.0$. These results suggest that $\mathrm{LVH}$ is the only factor contributing to microvascular dysfunction that would adversely affect the diagnostic accuracy of CFVR at a cut off value of $<2.0$ in a general population. In these patients we found a cut off value of CFVR $<1.5$ to be optimum when LVH is present, keeping in mind that there would be some loss of sensitivity. Further study is needed to validate the usefulness of this new cut off value in this subset of patients.

Several factors influence the decrease in the value of CFVR as previously reported. ${ }^{18}{ }^{29-31}$ In the current study, we showed that the presence of $\mathrm{LVH}$, the increase in resting heart rate, and aging decreased the value of CFVR in the LAD except for the presence of LAD stenosis with multivariate analysis. In patients with LVH or increased resting heart rate, the baseline coronary flow velocity increases because of the increase in myocardial oxygen consumption compared with normal subjects. $^{31}$ Moreover, coronary flow velocity during hyperaemia is reduced because of the functional and anatomical abnormality of the coronary microcirculation in patients with LVH. Therefore, coronary flow reserve is impaired in patients with LVH or increasing heart rate. Elderly patients have also been reported to have impaired endothelial function compared with young normal subjects. ${ }^{32}$ This may partly have affected the decrease in CFVR in elderly patients in this study.

\section{Study limitations}

Some methodological limitations of this study should be addressed. Firstly, some medications for the treatment of hypertension or hypercholesterolaemia are known to have a beneficial impact on the coronary circulation and thus favourably affect CFVR measurements. We did not discontinue any patient's medications, which may have affected our results. Secondly, although we tried to minimise the angle of incidence between the Doppler beam and coronary flow

Table 5 Multiple linear regression analysis with the value of CFVR in the LAD as the dependent variable $(r=0.631, p<0.0001)$

\begin{tabular}{lllll}
\hline & B & $95 \%$ Cl of $\mathbf{B}$ & $\boldsymbol{\beta}$ & p Value \\
\hline LAD stenosis $>50 \%$ & -1.070 & -1.242 to -0.898 & -0.570 & $<0.0001$ \\
Heart rate at rest & -0.014 & -0.021 to -0.007 & -0.181 & 0.0002 \\
LVH & -0.322 & -0.535 to -0.108 & -0.139 & 0.0033 \\
Age & -0.009 & -0.018 to -0.001 & -0.103 & 0.0384 \\
Diabetes & -0.129 & -0.535 to 0.108 & -0.072 & 0.1739 \\
SBP at rest & -0.003 & -0.006 to 0.001 & -0.064 & 0.2120 \\
Smoking & -0.075 & -0.253 to 0.102 & -0.040 & 0.4038 \\
Hypertension & 0.062 & -0.137 to 0.262 & 0.034 & 0.5395 \\
ACE inhibitor or ARB & -0.039 & -0.206 to 0.129 & -0.022 & 0.6495 \\
Hypercholesterolaemia & 0.029 & -0.142 to 0.200 & 0.016 & 0.7415 \\
Nitrate & -0.024 & -0.194 to 0.147 & -0.013 & 0.7826 \\
Calcium inhibitor & 0.012 & -0.176 to 0.200 & 0.007 & 0.8992 \\
Myocardial infarct in the LAD & -0.011 & -0.209 to 0.188 & -0.005 & 0.9166 \\
Male sex & 0.006 & -0.181 to 0.192 & 0.003 & 0.9537 \\
\hline
\end{tabular}

$\mathrm{ACE}$, angiotensin converting enzyme; $\mathrm{ARB}$, angiotensin II receptor blocker; $\mathrm{B}$, unstandardised regression coefficient; $\beta$, standardised coefficient; $\mathrm{Cl}$, confidence interval; $\mathrm{LVH}$, left ventricular hypertrophy; SBP, systolic blood pressure. 
direction, we did not correct the angle in the Doppler measurement. Hence, we may have underestimated the true velocity. However, for the purpose of CFVR measurement, the absolute velocity value is less important, as CFVR is the quotient of two velocities. Thirdly, coronary angiography has its own limitations when used to diagnose the severity of coronary artery disease. Any impact of these confounding factors would affect the groups equally.

\section{Conclusions}

The diagnostic accuracy for the detection of significant LAD stenosis when CFVR is $<2.0$ as determined by TTDE is high in a heterogeneous and high risk group of patients. Of the six major determinants of microvascular function only the presence of LVH significantly affects CFVR. On the basis of the current study, cut off values should be lowered to $<1.5$ to address this phenomenon and to maintain the high specificity of this test.

\section{ACKNOWLEDGEMENTS}

We acknowledge Dr Joseph Lodato, University of Chicago Medical Center, for his useful comments and suggestion.

\section{Authors' affiliations}

H Yoshitani, M Takeuchi, K Sakamoto, Department of Internal Medicine, Tane General Hospital, Osaka, Japan

T Akasaka, K Yoshida, Department of Internal Medicine and Cardiology, Kawasaki Medical School, Kurashiki, Japan

J Yoshikawa, Department of Internal Medicine and Cardiology, Graduate School of Medicine, Osaka City University, Osaka, Japan

There was no financial support of this manuscript.

\section{REFERENCES}

1 White CW, Wright CB, Doty DB, et al. Does visual interpretation of the coronary arteriogram predict the physiologic importance of a coronary stenosis? N Engl J Med 1984;310:819-24.

2 Arnett EN, Isner JM, Redwood DR, et al. Coronary artery narrowing in coronary heart disease: comparison of cineangiographic and necropsy findings. Ann Intern Med 1979;91:350-6.

3 Zir LM, Miller SW, Dinsmore RE, et al. Interobserver variability in coronary angiography. Circulation 1978;53:627-32.

4 Doucette JW, Corl PD, Payne HM, et al. Validation of a Doppler guide wire for intravascular measurement of coronary artery flow velocity. Circulation 1992:85:1899-911.

5 Segal J, Kern MJ, Scott NA, et al. Alterations of phasic coronary flow velocity in humans during percutaneous coronary angiography. J Am Coll Cardiol 1992;20:276-86.

6 Yoshida K, Yoshikawa J, Hozumi T, et al. Value of transesophageal color Doppler echocardiography in the evaluation of coronary artery anatomy and blood flow. Jpn Circ J 1990;54:298-303.

7 Iliceto S, Marangelli V, Memmola C, et al. Transesophageal Doppler echocardiographic evaluation of coronary blood flow velocity in baseline conditions and during dipyridamole-induced coronary vasodilation. Circulation 1991;83:61-9.

8 Redberg RF, Sobol Y, Chou TM, et al. Adenosine-induced coronary vasodilation during transesophageal Doppler echocardiography: rapid and safe measurement of coronary flow reserve ratio can predict significant left anterior descending coronary stenosis. Circulation 1995;92:190-6.

9 Demer LL, Gould KL, Goldstein RA, et al. Assessment of coronary artery disease severity by positron emission tomography: comparison with quantitative arteriography in 193 patients. Circulation 1989;79:825-35.

10 Hozumi T, Yoshida K, Ogata Y, et al. Noninvasive assessment of significant left anterior descending coronary artery stenosis by coronary flow velocity reserve with transthoracic color Doppler echocardiography. Circulation 1998;97:1557-62.

11 Hozumi T, Yoshida K, Akasaka T, et al. Noninvasive assessment of coronary flow velocity and coronary flow velocity reserve in the left anterior descending coronary artery by Doppler echocardiography: comparison with invasive technique. J Am Coll Cardiol 1998;32:1251-9.

12 Caiati C, Montaldo C, Zedda N, et al. Validation of a new noninvasive method (contrast-enhanced transthoracic second harmonic echo Doppler) for the evaluation of coronary flow reserve. J Am Coll Cardiol 1999:34:1193-200.

13 Pizzuto F, Voci P, Mariano E, et al. Assessment of flow velocity reserve by transthoracic Doppler echocardiography and venous adenosine infusion before and after left anterior descending coronary artery stenting. J Am Coll Cardiol 2001;38:155-62.

14 Yokoyama I, Momomura S, Ohtake T, et al. Reduced myocardial flow reserve in non-insulin-dependent diabetes mellitus. J Am Coll Cardiol 1997;30:1472-7

15 Nahser PJ Jr, Brown RE, Oskarsson H, et al. Maximal coronary flow reserve and metabolic coronary vasodilation in patients with diabetes mellitus. Circulation 1995;91:635-40.

16 Di Bello V, Pedrinelli R, Giorgi D, et al. Coronary microcirculation in essential hypertension: a quantitative myocardial contrast echocardiographic approach. Eur J Echocardiogr 2002;3:117-27.

17 Tanaka T, Oka Y, Tawara l, et al. Acute effects of nicotine content in cigarettes on coronary flow velocity reserve in men. Am J Cardiol 1998;82:1275-8.

18 Otsuka R, Watanabe H, Hirata K, et al. Acute effects of passive smoking on the coronary circulation in healthy young adults. JAMA 2001;286:436-41.

19 Memmola C, lliceto S, Napoli VF, et al. Coronary flow dynamics and reserve assessed by transesophageal echocardiography in obstructive hypertrophic cardiomyopathy. Am J Cardiol 1994;74:1147-51.

20 Hozumi T, Eisenberg M, Sugioka K, et al. Change in coronary flow reserve on transthoracic Doppler echocardiography after a single high-fat meal in young healthy men. Ann Intern Med 2002;136:523-8.

21 Hildick-Smith DJR, Shapiro LM. Potential use of transthoracic echocardiography in the assessment of coronary flow reserve. J Am Soc Echocardiogr 1999; 12:590-5.

22 Daimon $\mathbf{M}$, Watanabe $\mathrm{H}$, Yamagishi $\mathrm{H}$, et al. Physiologic assessment of coronary artery stenosis by coronary flow reserve measurements with transthoracic Doppler echocardiography: comparison with exercise thallium201 single-photon emission computed tomography. J Am Coll Cardiol 2001;37:1310-5.

23 Ruscazio M, Montisci R, Colonna $\mathrm{P}$, et al. Detection of coronary restenosis after coronary angioplasty by contrast-enhanced transthoracic echocardiographic Doppler assessment of coronary flow velocity reserve. J Am Coll Cardiol 2002;40:896-903.

24 Matsumura $\mathrm{Y}$, Hozumi T. Watanabe $\mathrm{H}$, et al. Cut-off value of coronary flow velocity reserve by transthoracic Doppler echocardiography for diagnosis of significant left anterior descending artery stenosis in patients with coronary risk factors. Am J Cardiol 2003;92:1389-93.

25 Hamouda MS, Kassem HK, Salama M, et al. Evaluation of coronary flow reserve in hypertensive patients by dipyridamole transesophageal Doppler echocardiography. Am J Cardiol 2000;86:305-8.

26 Miyazaki C, Takeuchi M, Yoshitani H, et al. Optimum hypoglycemic therapy can improve coronary velocity reserve in diabetic patients: demonstration by transthoracic Doppler echocardiography. Circ J 2003;67:945-50.

27 Rigo F, Richieri M, Pasanisi E, et al. Usefulness of coronary flow reserve over regional wall motion when added to dual-imaging dipyridamole echocardiography. Am J Cardiol 2003;91:269-73.

28 Lowenstein J, Tiano C, Marques G, et al. Simultaneous analysis of wall motion and coronary flow reserve of the left anterior descending coronary artery by transthoracic Doppler echocardiography during dipyridamole stress echocardiography. J Am Soc Echocardiogr 2003;16:607-13.

29 Galderisi M, Cicala S, Caso P, et al. Coronary flow reserve and myocardial diastolic dysfunction in arterial hypertension. Am J Cardiol 2002;90:860-4.

30 Krams R, Ten Cate FJ, Carlier SG, et al. Diastolic coronary vascular reserve: a new index to detect changes in the coronary microcirculation in hypertrophic cardiomyopathy. J Am Coll Cardiol 2004;43:670-7.

31 McGinn AL, White CW, Wilson RF. Interstudy variability of coronary flow reserve; influence of heart rate, arterial pressure, and ventricular preload. Circulation 1990;81:1319-30

32 Tao J, Jin YF, Young Z, et al. Reduced arterial elasticity is associated with endothelial dysfunction in persons of advancing age: comparative study of noninvasive pulse wave analysis and laser Doppler blood flow measurement. Am J Hypertens 2004; 17:654-9. 